\title{
A AGRICULTURA DE PRECISÃO PARA A GESTÃO ECONÔMICA E SUSTENTABILIDADE AMBIENTAL DA CAFEICULTURA
}

\author{
Leandro da Silva Almeida ${ }^{1}$, Ednaldo Carvalho Guimarães ${ }^{2}$ \\ ${ }^{1}$ Doutorando em Agronomia, Instituto de Ciências Agrárias, Universidade Federal de Uberlândia (UFU), \\ Uberlândia, Minas Gerais. E-mail: almeidalean26@gmail.com \\ ${ }^{2}$ Professor Titular, Faculdade de Matemática, Universidade Federal de Uberlândia (UFU), Uberlândia, Minas \\ Gerais.
}

RESUMO: A elaboração de mapas de fertilidade do solo permite que sejam aplicados os nutrientes de acordo com a necessidade pontual, assim melhorando a eficiência da aplicação. O objetivo deste trabalho foi modelar a dependência espacial do $\mathrm{pH}$ e da saturação de bases (V) do solo, gerando os mapas de fertilidade e verificar as vantagens econômicas e ambientais da agricultura de precisão na atividade cafeeira. $\mathrm{O}$ experimento foi realizado na Fazenda Brasil que possui 65 ha de cafeeiro arábica (Coffea arabica L.), localizada no município de Araguari-MG, utilizando uma malha de um ponto por hectare, totalizando 65 pontos. Foram ajustados os semivariogramas para o $\mathrm{pH}$ e a saturação de bases $(\mathrm{V})$, ambos apresentaram forte dependência espacial o modelo de semivariograma ajustado foi o gaussiano. Os dados foram interpolados por krigagem ordinária. $\mathrm{O}$ uso da agricultura de precisão na área em estudo representa uma economia de 30\% na aplicação de calcário, além disso, a agricultura de precisão contribuirá para a sustentabilidade ambiental da cafeicultura já que o nutriente seria aplicado de acordo com a demanda localizada, evitando excesso e escassez dentro da área.

Palavras-chave: Cafeicultura sustentável. Geoestatística. Cafeicultura de precisão. Gestão da cafeicultura.

\section{AGRICULTURE OF PRECISION FOR THE ECONOMY MANAGEMENT AND ENVIRONMENTAL SUSTAINABILITY COFFEE GROWING}

\begin{abstract}
The development of soil fertility maps allows the nutrients to be applied according to the need, thus improving the efficiency of the application. The objective of this work was to model the spatial dependence of $\mathrm{pH}$ and soil saturation $(\mathrm{V})$, generating fertility maps and verifying the economic and environmental advantages of precision agriculture in coffee production. The experiment was carried out at Fazenda Brasil, which has 65 ha of arabica coffee (Coffea arabica L.), located in the municipality of Araguari-MG, using a mesh of one point per hectare, totaling 65 points. Semivariograms were adjusted for $\mathrm{pH}$ and base saturation $(\mathrm{V})$, both presented a strong spatial dependence, the adjusted semivariogram model was Gaussian. The data were interpolated by ordinary kriging. The use of precision agriculture in the study area represents a 30\% saving in the application of limestone. In addition, precision farming will contribute to the environmental sustainability of coffee
\end{abstract}

Cultura Agronômica, Ilha Solteira, v.25, n.4, p.431-440, 2016 
production as the nutrient would be applied according to the localized demand, avoiding excess and shortage in the area.

Key words: Sustainable coffee cultivation. Geostatistics. Precision coffee makers. Management of coffee cultivation.

\section{INTRODUÇÃO}

A importância da cafeicultura no contexto econômico e ambiental brasileiro está na sua magnitude, pois ocupa uma área de mais de 2,2 milhões de hectares plantados no país, sendo que destes 78,5 \% são de cafeeiro arábica (CONAB, 2016). O Brasil no ano de 2015 movimentou com a exportação de café mais de US\$ 5.555 milhões, sendo que o café foi o terceiro produto agrícola nos valores de exportação, ficando atrás apenas da carne de frango e do complexo-soja, segundo os dados da Secretaria de Comércio Exterior do Ministério do Desenvolvimento, Indústria e Comércio Exterior (2015). Para ressaltar a importância da cafeicultura no contexto ambiental, a sua área é maior que todo o território do estado Sergipe, que possui 2.1 milhões de hectares, por exemplo.

$\mathrm{O}$ cafeeiro é exigente em nutrientes, sendo o cálcio $(\mathrm{Ca})$ e o magnésio $(\mathrm{Mg})$ normalmente fornecidos por corretivos da acidez do solo. A calagem traz inegáveis benefícios ao cafeeiro, dentre os quais se destacam o fornecimento de $\mathrm{Ca}$ e $\mathrm{Mg}$, a elevação do $\mathrm{pH}$ à valores ideais entre 5,5 e 6,5 (ALVAREZ V. et al., 1999), com a consequente diminuição da toxidez de alumínio (Al), manganês ( $\mathrm{Mn}$ ) e ferro (Fe), o aumento da disponibilidade de fósforo (P) e também da atividade microbiológica do solo (CORRÊA et al., 2007). Tendo em vista que os solos de fronteiras agrícolas para a cultura do café, em sua maioria, apresentam acidez elevada, baixa capacidade de retenção de cátions (CTC) e baixos teores de bases trocáveis, como Ca e Mg e frequentemente de enxofre (S) (TOMAZ et al., 2003), o manejo eficiente dos nutrientes é de grande importância na gestão da propriedade.

Assim a elaboração dos mapas de fertilidade da área de cultivo permite que sejam aplicados os elementos (nutrientes) de acordo com a necessidade quase pontual e não mais uniformemente como é realizado convencionalmente pelos produtores (BERNADI et al., 2014). Com o manejo específico, procura-se minimizar as perdas de insumos, otimizando o sistema de produção. Na agricultura de precisão, o manejo sítio-específico surge em contraposição à visão tradicional que preconiza manejo baseado nas condições médias da lavoura, o que implica na adoção de tratamentos diferenciados, segundo variações na área de cultivo, diminuindo a possibilidade de subdosagens ou superdosagens de fertilizantes, acarretando em condições adversas de desenvolvimento da cultura, além de maiores possibilidades de perdas de nutrientes e poluição de águas subterrâneas e superficiais (HURTADO et al., 2008).

Cultura Agronômica, Ilha Solteira, v.25, n.4, p.431-440, 2016 
A produtividade das culturas varia espacialmente, e determinar as causas dessas variações é o desafio que enfrenta a agricultura de precisão. As variações espaciais podem ser estudadas por meio de diferentes métodos estatísticos, que permitem elaborar mapas e delimitar áreas de manejo diferenciadas (FARIAS et al., 2003). Embora esses métodos estatísticos permitam inferir sobre a variabilidade do solo (vertical e horizontal), a dependência espacial entre as amostras somente pode ser modelada por meio de análises geoestatísticas (MANZIONE; ZIMBACK, 2011).

A agricultura de precisão busca entender estas variações por meio da quantificação da variabilidade espacial e temporal dos fatores ligados às interações solo-água-planta, visando otimizar o uso de insumos agrícolas, realizando a distribuição adequada, em cada setor do campo de produção. $O$ conceito de agricultura de precisão apesar de ser simples é uma prática muito complexa, que exige conhecimentos de sensoriamento, manipulação de dados, controle, localização e outras informações geográficas (MOLIN, 1997).

A agricultura de precisão pode e deve se tornar grande aliada da gestão das propriedades rurais, tanto da gestão econômica, como, principalmente, da gestão ambiental, pois permite a produção de alimentos e outros produtos de alta qualidade por meio do uso racional dos recursos naturais e dos insumos agrícolas, podendo, assim, assegurar uma produção sustentável (FARIAS et al., 2003).

Assim o objetivo deste trabalho foi modelar a dependência espacial do $\mathrm{pH}$ e da saturação de bases (V) da área de cultivo e comprovar as vantagens econômicas e ambientais da agricultura de precisão na cafeicultura em uma fazenda do cerrado mineiro.

\section{MATERIAL E MÉTODOS}

O trabalho foi desenvolvido na fazenda Brasil, pertencente ao produtor Ladmir de Lima, localizada no município de Araguari-MG, cujas coordenadas geográficas são: 18³2'55.99"S, $48^{\circ} 24^{\prime} 10.46^{\prime}$ "O. A altitude é de 940 metros. A propriedade possui 65 hectares cultivados com cafeeiro da espécie arábica (Coffea arabica L.), da variedade Mundo Novo, plantados em 1989, cultivados em um Latossolo Vermelho Amarelo (38\% de argila em média). Toda a lavoura cafeeira é irrigada por gotejamento, sendo ainda que a propriedade adota técnicas de fertirrigação e quimigação. A Fazenda conta ainda com certificações como o Certifica Minas ${ }^{\circledR}$ e a Utz Certified ${ }^{\circledR}$, sendo assim comprometida com o manejo sustentável.

Foram coletadas amostras de solo de 0 a $20 \mathrm{~cm}$ de profundidade, na projeção da copa do cafeeiro conforme Cantarutti et al. (1999). Todas as amostras coletadas foram georeferenciadas. A malha utilizada foi de 1 hectare (FAULIM et al., 2004), totalizando 65 pontos.

Foram determinados o pH e a saturação de bases do solo de acordo com as análises de rotinas do laboratório de análise de solo da Universidade Federal de Uberlândia. 
Com os dados organizados, as análises dos resultados foram procedidas com o auxílio do programa $\mathrm{R}$ versão 3.2.1, utilizando o pacote de dados geoR (RIBEIRO JÚNIOR; DIGGLE, 2001).

Foram realizadas as estatísticas descritivas de todos os atributos, visando conhecer o comportamento geral dos dados, e verificar possíveis valores atípicos que pudessem comprometer as análises seguintes. Para cada atributo do solo, foram gerados os gráficos de box-plots a fim de identificar possíveis valores de outlines de cada atributo na área.

Foram realizadas as análises de tendência dos dados, por meio de gráficos de dispersão para as direções leste (x) e norte (y) de cada atributo do solo, os mapas de localização dos pontos amostrais na área, e os histogramas de todos os atributos analisados, a fim de verificar o comportamento da variável na área e a assimetria ou simetria dos dados respectivamente, que pode interferir na modelagem espacial e no processo de interpolação pela krigagem (YAMAMOTO; LAMDIM, 2013).

No ajuste de semivariogramas foram avaliados os modelos, por meio da técnica de validação cruzada, sendo selecionados aqueles que melhor descreveram o comportamento espacial dos fatores (NANNI et al., 2011; YAMAMOTO; LANDIM, 2013). Após estas verificações para os atributos que possuíram dependência espacial, aplicou-se a krigagem ordinária para a interpolação (YAMAMOTO; LANDIM, 2013; SOARES, 2006; VIEIRA et al., 1983).

Para a divisão das faixas de manejo, obtidas nos mapas temáticos, foram utilizados os critérios relativos a cada atributo do solo conforme proposto por Alvarez V. et al. (1999), no Manual de Recomendações do Uso de Fertilizantes e Corretivos em Minas Gerais ( $5^{\mathrm{a}}$ Aproximação).

\section{RESULTADOS E DISCUSSÃO}

O valor médio do $\mathrm{pH}$, em água do solo foi de 5,8 sendo classificado como um nível bom agronomicamente. Entretanto, o valor máximo de 6,78 já é considerado um pH alto, nível no qual haverá deficiência de micronutrientes, e o valor mínimo de 5,14 já é considerado baixo, onde há presença de alumínio tóxico nas raízes do cafeeiro (VENDRAME et al., 2007). Carneiro et al. (2009), estudando os solos do cerrado sobre diferentes manejos, concluíram que o solo sofre interferência direta do manejo em suas características físico-químicas.

A saturação de bases (V) do solo média foi de 53,07, oscilando entre 24,74 a 69,05 com uma variância de 95,7, observa-se que, da mesma forma que o $\mathrm{pH}$, existem faixas com ótima saturação entretanto há faixas com baixa saturação. De acordo com Alvarez V. et al. (1999), o valor ideal de saturação de bases (V) para o cafeeiro é de $60 \%$, ainda reforçam que a necessidade de correção quando a saturação estiver abaixo de 50\%. Corrêa et al. (2007), estudando o valor ideal de saturação de bases do solo, observaram também que a

Cultura Agronômica, Ilha Solteira, v.25, n.4, p.431-440, 2016 
produtividade do cafeeiro é crescente até $60 \%$ de saturação de bases (V), e decrescente rapidamente após o índice de $60 \%$ de saturação de bases (V).

Nas Figuras 1 e 2, são apresentados para os dois atributos do solo estudados, os semivariogramas ajustados, por meio da técnica de validação cruzada e selecionados aqueles que apresentaram melhores parâmetros quando avaliados por meio de validação cruzada (VIEIRA et al., 1983; YAMAMOTO; LAMDIM, 2013).

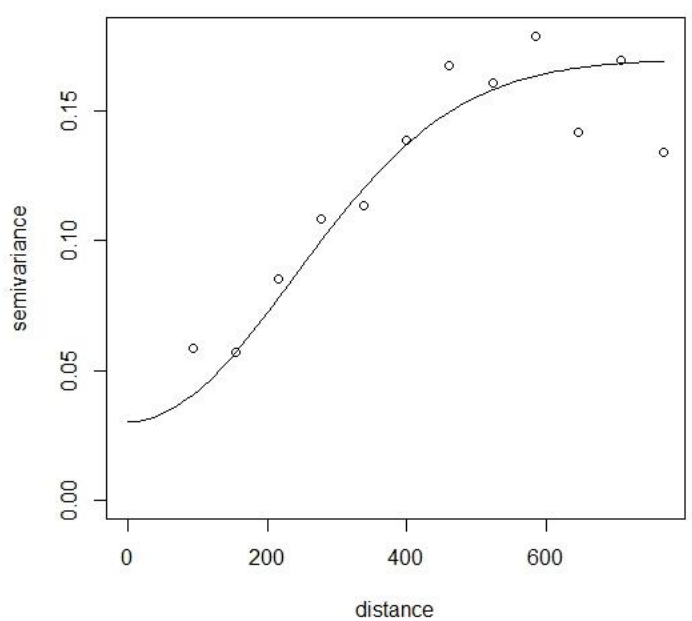

Figura 1. Semivariograma ajustado do $\mathrm{pH}$.

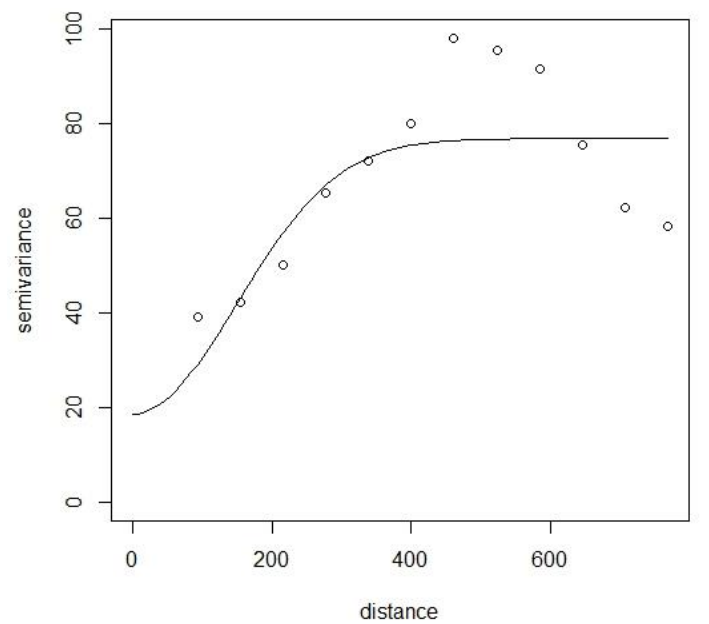

Figura 2. Semivariograma ajustado da saturação de bases.

$\mathrm{Na}$ Tabela 1, são apresentados os modelos de semivariogramas ajustados para os atributos do solo, os parâmetros do ajuste e o grau de dependência espacial segundo Cambardella et al. (1994).

Tabela 1. Modelos, parâmetros, relação $\mathrm{Co} / \mathrm{Co}+\mathrm{C}$ e grau da dependência espacial dos semivariogramas ajustados para o pH e a saturação e bases.

\begin{tabular}{|c|c|c|c|c|c|c|}
\hline Atributo & Modelo & $\mathrm{C}$ & $\mathrm{CO}$ & Alcance & $\mathrm{Co} / \mathrm{Co}+\mathrm{C}^{1}$ & $\mathbf{G D}^{2}$ \\
\hline $\mathrm{pH}$ & Gaussiano & 0,14 & 0,03 & 575,99 & 0,18 & Forte \\
\hline V & Gaussiano & 58,33 & 18,56 & 359,73 & 0,24 & Forte \\
\hline
\end{tabular}

Observa-se na Tabela 1, que os atributos do solo pH e saturação de bases (V) apresentaram forte grau de dependência espacial (GD <0,25). Silva et al. (2008), também encontraram forte dependência espacial para o $\mathrm{pH}$ do solo cultivado com cafeeiro, entretanto o modelo ajustado foi o esférico. Já Reichert et al. (2008), encontraram também forte dependência espacial para a saturação de bases e moderada para o $\mathrm{pH}$ do solo, ajustado naquela oportunidade pelo modelo gaussiano para a saturação de bases e exponencial para o $\mathrm{pH}$. 
Observe que, o alcance real máximo do $\mathrm{pH}$ foi de 575,99 utm, que é 216,26 utm maior do que o alcance da saturação de bases, mesmo assim, com 379,73 utm a saturação de bases tem um ótimo alcance, já que a distância mínima entre os pontos amostrados foi de 77 utm. Baixos valores de alcance podem influir negativamente na qualidade das estimativas, uma vez que poucos pontos são usados para realização da interpolação na estimativa de valores em locais não medidos (LIMA et al., 2010).

Nas figuras 3 e 4 são apresentados os mapas temáticos de distribuição do pH e da V de bases na área.

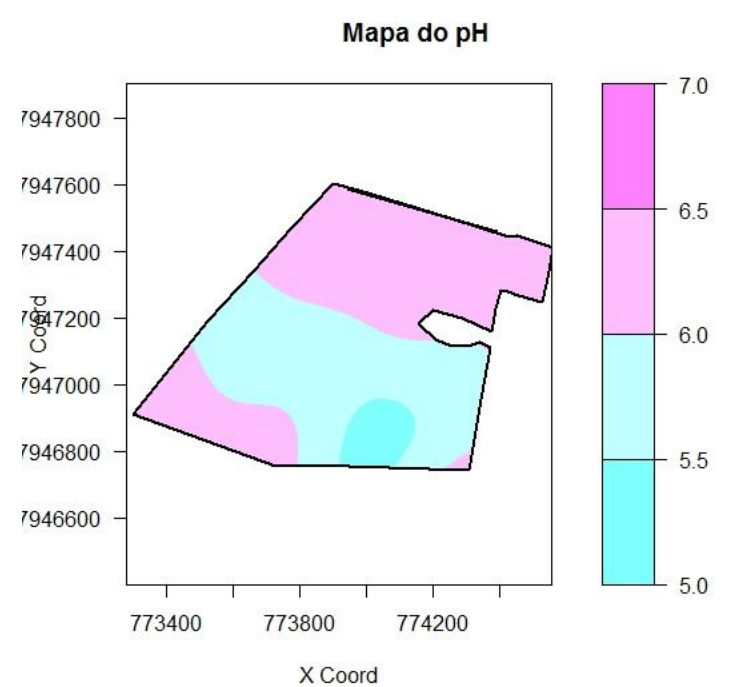

Figura 3. Mapa temático de distribuição do pH na área.

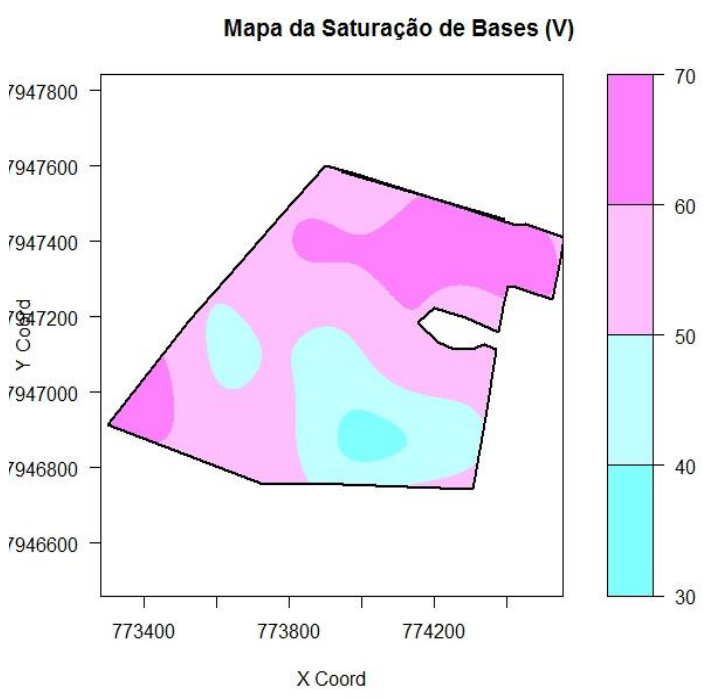

Figura 4. Mapa temático da distribuição espacial da V na área.

No mapa temático do $\mathrm{pH}$ (Figura 3), pode ser observado uma pequena faixa azul mais escuro, onde há necessidade de correção do solo no ano de $2015(\mathrm{pH}<5,5)$, o mesmo mapa apresenta uma faixa central (azul mais claro) de pH entre 5,5 e 6,0 que a principio, não há necessidade de correção neste ano de 2015, já que o alumínio $\left(\mathrm{Al}^{+3}\right)$ nesta faixa de pH está neutralizado, mas entretanto é uma zona de monitoramento mais constante, já que o pH está mais próximo do limite de intervenção ( $\mathrm{pH}<5,5)$ (ALVAREZ V. et al., 1999).

A saturação de bases (V) ideal do cafeeiro é de 60\%; observa-se no mapa (Figura 4) que a faixa em rosa mais escuro, pode ser considerada ideal (50-60\%); as demais partes estão acima ou abaixo do ideal, sendo que a faixa em rosa mais claro está acima do ideal $(>60 \%)$, nível no qual a produção decresce rapidamente (CORRÊA et al., 2007). O restante, as machas em azul que se encontram abaixo de $50 \%$ de $\mathrm{V}$, que seriam faixas com maior necessidade de correção (CORRÊA et al., 2007; ALVAREZ V. et al., 1999).

A correção do solo do cafeeiro, recomenda em Minas Gerais, é para se elevar o pH para acima de 5,5 e elevar a saturação de bases (V) para 60\%; no solo em estudo a saturação de bases média está em 53,07\%. Se o produtor optar pela agricultura convencional ele terá uma necessidade de calcário de aproximadamente $430 \mathrm{~kg}$ por hectare, cálculo feito utilizando-se o 
método de saturação de bases (ALVAREZ V. et al., 1999). Entretanto, observa-se na Figura 4 (saturação de bases), que o solo possui uma faixa acima de $60 \%$ de $\mathrm{V}$, e possui uma grande mancha abaixo de $50 \%$ de V, chegando ao valor mínimo de $30 \%$ de V (Figura 4).

Diante do constatado surgem dois questionamentos: a) a agricultura convencional está contribuindo para uma maior eficiência de gestão desta propriedade? b) e o que isto tem a ver com sustentabilidade? As respostas a estes questionamentos são relativamente claras quando observa-se que se aplicar $430 \mathrm{~kg}$ de calcário na área acima de 60\% de saturação, estará havendo um excedente, ou seja, custos desnecessário e ocorrência de impacto ambiental. Por outro lado, ao se aplicar a mesma dose nas áreas abaixo de 50\%, principalmente a faixa abaixo de $40 \%$ de $\mathrm{V}$, não haverá total correção de solo e, com isso, menor crescimento das raízes das plantas, ou seja, além de não se alcançar o potencial produtivo da planta, pode-se haver perdas ambientais, pela não capacidade da planta em absorver outros nutrientes, que dependem desta correção.

Se optar por usar a média para corrigir o solo da área, com o calcário supracitado, seriam necessárias 32 toneladas para fazer a correção do solo na área. Se a correção for realizada por faixa, aplicando-se as doses necessárias, observa-se que a primeira faixa, em azul mais escuro (faixa I), com uma necessidade média de calcário de 1,8 toneladas por hectare; a outra faixa, em azul um pouco mais claro (faixa II), necessita em média de 1,03 toneladas deste calcário; e na terceira faixa (faixa III), essa faixa é representada na Figura 4, pela cor rosa claro, seria necessário 0,32 toneladas do referido calcário por hectare. Esses seguimentos representam aproximadamente 3,5\%,22,5\% e 72,5\% da área, ou seja, seria necessário para corrigir a faixa I: 4 toneladas; a faixa II: 15 toneladas; e a faixa III: 3,5 toneladas. Totalizando, 22,5 toneladas de calcário, número $30 \%$ menor que quando se faz a correção pela média.

Além disso, a aplicação de calcário nas faixas I, II, quando se utiliza a média é $70 \%$ e $52 \%$ menor respectivamente que a dose realmente necessária para corrigir essas faixas; já na faixa III, a aplicação pela média é 34\% maior que a quantidade realmente necessária; na última faixa (rosa mais escuro), fica claro que toda a correção do solo é desnecessária. Esses números expressam a grande importância do uso de ferramenta como a geoestatística para gerir a aplicação de insumos nas propriedades, corroborando com os resultados obtidos por Almeida e Guimarães (2016), que verificaram também que o uso da técnica de geoestatística, permite uma melhor gestão dos insumos.

\section{CONCLUSÃO}

$\mathrm{O}$ pH e a saturação de bases do solo (V) apresentaram forte dependência espacial, com alcance de 575,99 utm e 359,73 utm respectivamente.

O uso da agricultura de precisão na área em estudo representa uma economia de 30\% na aplicação de calcário.

Cultura Agronômica, Ilha Solteira, v.25, n.4, p.431-440, 2016 
A agricultura de precisão contribuirá para a sustentabilidade ambiental da cafeicultura já que o nutriente seria aplicado de acordo com a demanda localizada.

\section{REFERÊNCIAS BIBLIOGRÁFICAS}

ALMEIDA, L. S., GUIMARÃES, E. C. Geoestatística e análise fatorial exploratória para representação espacial de atributos químicos do solo, na cafeicultura. Coffee Science, Lavras, v. 11, n. 2, p.195 - 203, 2016.

ALVAREZ V., V. H.; NOVAIS, R. F.; BARROS, N. F.; CANTARUTTI, R. B.; LOPES, A. F. Interpretação dos resultados das análises do solos. In: RIBEIRO, A. C., GUIMARÃES, P. T. G., ALVAREZ V., V. H. (Ed.). Recomendações para o uso de corretivos e fertilizantes em Minas Gerais - $5^{\text {a }}$ Aproximação. Viçosa: Comissão de Fertilidade do Solo do Estado de Minas Gerais, 1999. cap. 5, p. 25-32.

BERNADI, A. C. C. NAIME, J. M.; RESENDE, A. V.; BASSOI, L. H. Agricultura de precisão: resultados de um novo olhar. Embrapa: Brasília, 2014. 596 p.

CAMBARDELLA, C. A.; MOORMAM, T. B.; PARKIN, T. B.; NOVAK, J. M.; TURCO. R. F.; KONOPKA, A. E. Field-scale variability of soil proprieties in central Iowa soils. Soil Science Society America Journal, Medison, v. 58, n. 5, p.1240-1248, 1994.

CANTARUTTI, R. B.; AVAREZ V., V. H.; RIBEIRO, A. C. Amostragem do solo. In: RIBEIRO, A.C., GUIMARÃES, P.T.G., ALVAREZ V., V.H. (Ed.). Recomendações para o uso de corretivos e fertilizantes em Minas Gerais - $5^{\text {a }}$ Aproximação. Viçosa: Comissão de Fertilidade do Solo do Estado de Minas Gerais, 1999. cap. 3, p.13-20.

CARNEIRO, M. A. C.; SOUZA, E. D; REIS, E. F.; PEREIRA, H. S.; AZEVEDO, W. S. Atributos físicos, químicos e biológicos de solo de cerrado sob diferentes sistemas de uso e manejo. Revista Brasileira de Ciências do Solo, Viçosa, v. 33, n. 1, p.147-157, 2009.

COMPANHIA NACIONAL DE ABASTECIMENTO - CONAB. Estimativas de Safras Agrícolas. Levantamento Café. Disponível em: http://www.conab.gov.br/ . Acesso em: 06 jan. 2016.

CORRÊA, J. B. REIS, T. H. P.; POZZA, A. A. A.; GUIMARÃES, P. T. G.; CARVALHO, J. G. Índice de Saturação por Bases na nutrição e na produtividade de cafeeiros catuaí vermelho (Coffea arabica L.). Coffee Science, Lavras, v. 2, n. 2, p.159-167, 2007.

FAUliM, G. D. C.; MOLIN, J. P.; MAGALHÃES, R. P.; STANISLAVSKI, W. M. Estudo da espacialidade da maturação de café (Coffea arabica L.) visando a confecção de mapas de produtividade. In: CONGRESSO BRASILEIRO DE AGRICULTURA DE PRECISÃO CONBAP, 1., 2004, Piracicaba. Anais... Piracicaba: ESALQ/USP, 2004. CD-ROM.

FARIAS, P. R. S. NOCITI, L. A. S.; BARBOSA, J. C.; PERECIN, D. Agricultura de precisão: mapeamento da produtividade em pomares cítricos usando geoestatística. Revista Brasileira de Fruticultura, Jaboticabal, v. 25, n. 2, p.235-241, 2003.

Cultura Agronômica, Ilha Solteira, v.25, n.4, p.431-440, 2016 
HURTADO, S. M. C.; RESENDE, A. V.; SILVA, C. A.; CORAZZA, E. J.; SHIRATSUCHI, L. S. Agricultura de Precisão: possibilidade de manejo da adubação nitrogenada para o milho no cerrado. Planaltina: Embrapa Cerrados, 2008. 48 p. (Embrapa Cerrados, Boletim de Pesquisa e Desenvolvimento, 214).

LIMA, J. S. S.; SOUZA, G. S.; SILVA, S. A. Amostragem e variabilidade espacial de atributos químicos do solo em área de vegetação natural em regeneração. Revista Árvore, Viçosa, v. 34, n. 1, p.127-136, 2010.

MANZIONE, R. L.; ZIMBACK, C. R. L. Análise Espacial Multivariada Aplicada na Avaliação da Fertilidade do Solo. Engenharia na Agricultura, Viçosa, v. 19, n. 3, p.227$235,2011$.

MINISTÉRIO DO DESENVOLVIMENTO, INDÚSTRIA E COMERCIO EXTERIOR Secretária de Comércio Exterior, 2015. Prévia da balança comercial 2015. Disponível em: http://www.desenvolvimento.gov.br/sitio/interna/interna.php?area=5\&menu=1161. Acesso em: 08 jan. 2016.

MOLIN, J. P. Agricultura de Precisão. Parte 1. O que é e estado da arte em sensoriamento. Engenharia Agrícola, Jaboticabal, v. 17, n. 2, p.108-121, 1997.

NANNI, M. R.; POVH, S. P.; DEMATTÊ, J. A, M.; OLIVEIRA, R. B.; CHICATI, M. L.; CEZAR, E. Optimum size in grid soil sampling for variable rate application in site-specific management. Scientia Agricola, Piracicaba, v. 68, n. 3, p.386-392, 2011.

RIBEIRO JÚNIOR, P. J.; DIGGLE, P. J. geoR: a package for geostatistical analysis. RNEWS, Pelotas, v. 1, n. 2, p.15-18, 2001.

REICHERT, J. M. DARIVA, T. H.; REINERT, D. J.; SILVA, V. R. Variabilidade espacial de Planossolo e produtividade de soja em várzea sistematizada: análise geoestatística e análise de regressão. Ciência Rural, Santa Maria, v. 38, n. 4, p.981-988, 2008.

SILVA, F. M. SOUZA, Z. M.; FIGUEIREDO, C. A. P.; VIEIRA, L. H. S.; OLIVEIRA, E. Variabilidade espacial de atributos químicos e produtividade da cultura do café em duas safras agrícolas. Ciência Agrotecnologica, Lavras, v. 32, n. 1, p.231-241, 2008.

SOARES, A. Geoestatística para ciências da terra e do ambiente. 2. ed. Lisboa: IST Press, 2006. 2014 p.

TOMAZ, M. A.; SILVA, S. R.; SAKIYAMA, N. S.; MARTINEZ, H. E. P. Eficiência de absorção, translocação e uso de cálcio, magnésio e enxofre por mudas enxertadas de Coffea arabica. Revista Brasileira de Ciência do Solo, Viçosa, v. 27, n. 5, p.885-892, 2003.

VENDRAME, P. R. S. BRITO, O. R.; QUANTINI, C.; BECQUER, T. Disponibilidade de cobre, ferro, manganês e zinco em solos sob pastagens na Região do Cerrado. Pesquisa Agropecuária Brasileira, Brasília, v. 42, n. 6, p.859-864, 2007.

Cultura Agronômica, Ilha Solteira, v.25, n.4, p.431-440, 2016 
VIEIRA, S. R. HATFIELD, J. L.; NIELSEN, D. R.; BIGGAR, J. W. Geostatistical theory and application to variability of some agronomical properties. Hilgardia, Berkeley, v. 31, n. 3, p.1-75, 1983.

YAMAMOTO, J. K.; LANDIM, P. M. B. Geoestatística: conceitos e aplicações. São Paulo: Oficina Textos, 2013. 215 p. 